Original Article

\title{
Thoracoscopic Surgery for Pneumothorax Following Outpatient Drainage Therapy
}

\author{
Atsushi Sano, MD, PhD and Takuma Yotsumoto, MD
}

\begin{abstract}
Purpose: We investigated the outcomes of surgery for pneumothorax following outpatient drainage therapy.

Methods: We reviewed the records of 34 patients who underwent operations following outpatient drainage therapy with the Thoracic Vent at our hospital between December 2012 and September 2016. Indications for outpatient drainage therapy were pneumothorax without circulatory or respiratory failure and pleural effusion. Indications for surgical treatment were persistent air leakage and patient preference for surgery to prevent or reduce the incidence of recurrent pneumothorax.

Results: Intraoperatively, 9 of $\mathbf{3 4}$ cases showed loose adhesions around the Thoracic Vent, all of which were dissected bluntly. The preoperative drainage duration ranged from 5 to 13 days in patients with adhesions and from 3 to 19 days in those without adhesions, indicating no significant difference. The duration of preoperative drainage did not affect the incidence of adhesions. The operative duration ranged from 30 to 96 minutes in patients with adhesions and from 31 to 139 minutes in those without adhesions, also indicating no significant difference.

Conclusion: Outpatient drainage therapy with the Thoracic Vent was useful for spontaneous pneumothorax patients who underwent surgery, and drainage for less than 3 weeks did not affect intraoperative or postoperative outcomes.
\end{abstract}

Keywords: pneumothorax, drainage, outpatient, operation

\section{Introduction}

Pneumothorax is one of the common causes of acute chest pain, especially in young males. Chest drainage is sometimes necessary for the initial treatment of pneumothorax. Although chest drainage is performed mostly during hospitalization, it is applied on an outpatient basis for mild or moderate pneumothorax in some institutions. ${ }^{1-7)}$

Department of Thoracic Surgery, Chigasaki Municipal Hospital, Chigasaki, Kanagawa, Japan

Received: March 15, 2017; Accepted: April 15, 2017

Corresponding author: Atsushi Sano, MD, PhD. Department of Thoracic Surgery, Chigasaki Municipal Hospital, 5-15-1 Honson, Chigasaki, Kanagawa 253-0042, Japan

Email: sanoa-tky@umin.ac.jp

(C)2017 The Editorial Committee of Annals of Thoracic and Cardiovascular Surgery. All rights reserved.
Even after chest drainage, some patients require surgical intervention for prolonged air leakage or undergo bullectomy with the hope of preventing pneumothorax relapse. Thoracic surgeons at our hospital perform outpatient chest drainage using the Thoracic Vent (UreSil, Skokie, IL, USA) for mild or moderate pneumothorax, and use a thoracoscopic approach for patients who require surgery. ${ }^{1-3)}$ Although several papers have reported the outcomes of outpatient drainage for pneumothorax, there is minimal data on surgical outcomes following outpatient drainage. ${ }^{4)}$

Herein, we present the outcomes of surgery for pneumothorax following outpatient drainage therapy.

\section{Materials and Methods}

We reviewed the records of 34 patients who underwent operations following outpatient drainage therapy 


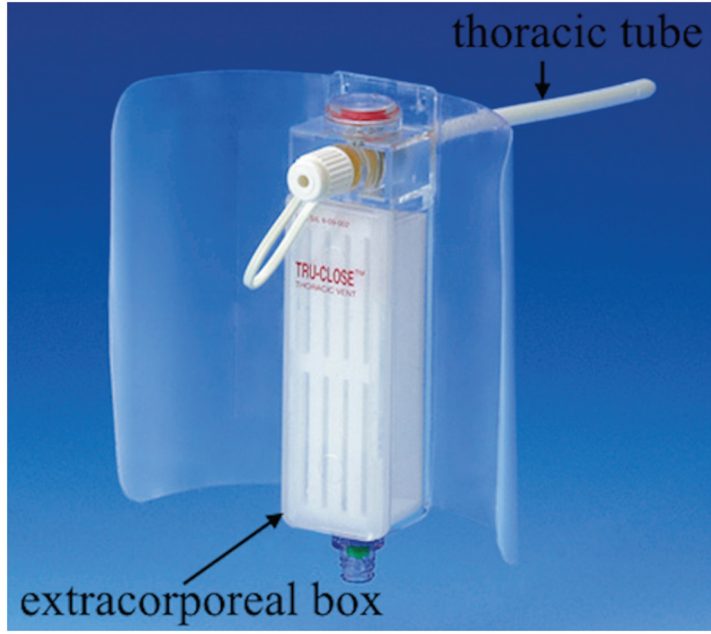

Fig. 1 Thoracic Vent.

with the Thoracic Vent in our hospital between December 2012 and September 2016. The institutional review board approved this study on April 15, 2013 (Project Approval No. 2013-05).

Indications for outpatient drainage therapy were pneumothorax without circulatory or respiratory failure and pleural effusion. Patients with severe pneumothorax were excluded because of the possibility of re-expansion pulmonary edema. Patients with pleural effusion were excluded because the Thoracic Vent has only a small chamber for drainage output. We included patients of any age with either primary or secondary pneumothorax. ${ }^{1)}$

The Thoracic Vent is a 10- or 13-Fr thoracic tube with a small extracorporeal box $(9 \times 2.5 \times 2 \mathrm{~cm})$ containing a one-way valve (Fig. 1).5,6) A 13-Fr tube is used at our institution. The Thoracic Vent is inserted under local anesthesia through the second intercostal space in the mid-axillary line. Patient condition is checked via chest $\mathrm{X}$-ray two or three times a week during outpatient drainage. ${ }^{2)}$ Taking a shower or bath is allowed as long as the Thoracic Vent is kept dry. We permit desk work and forbid manual labor to prevent dislocation of the Thoracic Vent. We do not change the drape during Thoracic Vent drainage.

The indications for surgical treatment were as follows: 1) persistent air leakage or 2) patient preference for surgery to prevent or reduce the incidence of recurrent pneumothorax. The decision to perform surgery was made within 1 week of pneumothorax. If air leakage was present at the first check-up after drainage began, we considered surgery. If leakage continued at 1 week after starting drainage, we generally decided upon and scheduled the operation. Details on the management of outpatient drainage therapy using the Thoracic Vent have been described previously. ${ }^{2)}$

All statistical analyses were performed with EZR (Saitama Medical Center, Jichi Medical University, Saitama, Japan), a graphical user interface for R (The R Foundation for Statistical Computing, Vienna, Austria). ${ }^{8}$ More precisely, it is a modified version of the $\mathrm{R}$ commander designed to add statistical functions frequently used in biostatistics. We used the Fisher's exact test for comparisons of categorical data between two groups, and the Mann-Whitney U-test to compare continuous data such as drainage duration and length of operation. We considered $p<0.05$ to indicate statistical significance.

\section{Results}

Of the 34 patients, $28(82 \%)$ were males and $6(18 \%)$ were females. Their ages ranged from 15 to 73 years, with a mean age of 35 years. Pneumothorax occurred on the right side in 16 patients and on the left side in 18 patients. No cases of synchronous bilateral pneumothorax were included. Surgery was performed for persistent air leakage in 15 of 34 patients (44\%), and for the prevention of relapse in 19 patients (56\%). The preoperative drainage duration ranged from 3 to 19 days, with a mean of 9 days. One patient was admitted 4 days preoperatively because he required negative pressure drainage due to massive air leakage. The remaining 33 patients were admitted one or two working days before the operation, as scheduled.

During the same period, 57 patients were treated for spontaneous pneumothorax with the Thoracic Vent only. Of these 57 patients, 10 underwent surgery for preventing recurrent pneumothorax after removal of the Thoracic Vent, and 11 experienced recurrent pneumothorax.

All patients underwent either thoracoscopic bullectomy or bulla ligation with polyglycolic acid sheet coverage. The intraoperatively inserted chest tube was removed a few days after the operation in all patients. The operation duration ranged from 30 to 139 minutes, with a mean duration of 58 minutes. There were no instances of lung injury or bleeding due to outpatient drainage.

In 9 of 34 cases, loose adhesions were observed around the Thoracic Vent intraoperatively, all of which were dissected bluntly (Fig. 2). Preoperative drainage duration ranged from 5 to 13 days (mean, 9 days) in patients with adhesions and from 3 to 19 days (mean, 8 days) in those without adhesions, indicating no significant difference ( $p=0.56$, Mann-Whitney U-test) 


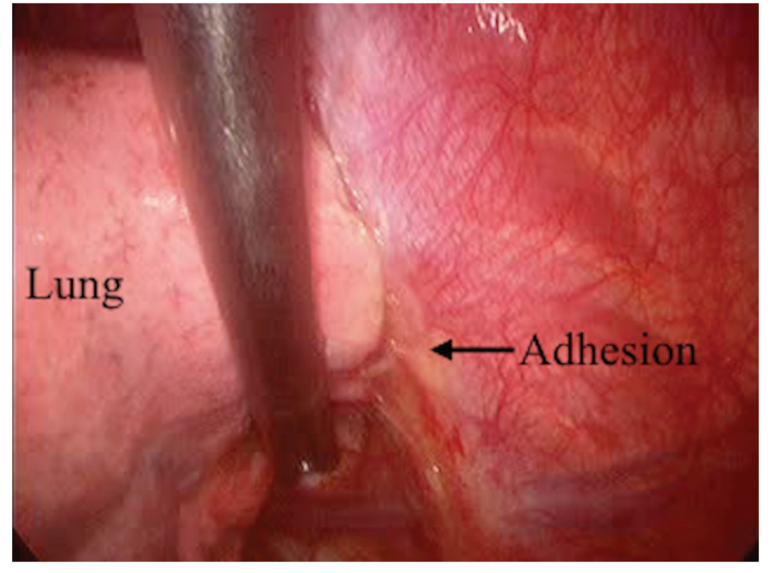

Fig. 2 Intraoperative view of a patient with adhesions. A linear adhesion along the Thoracic Vent is seen. The adhesion was easily dissected bluntly.

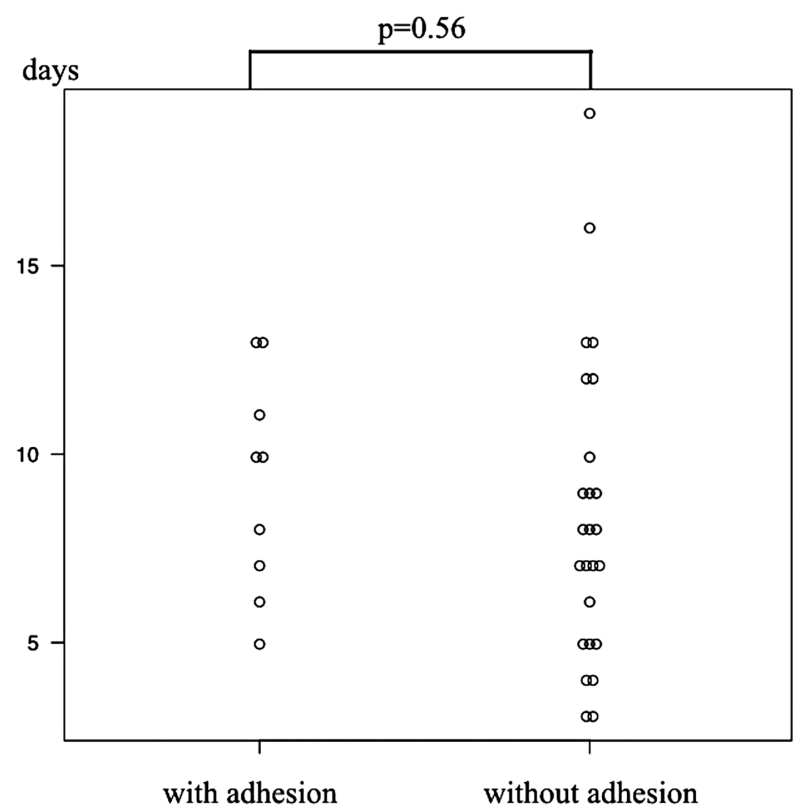

Fig. 3 Distribution of preoperative drainage duration. There is no statistically significant difference between patients with and without adhesions.

(Fig. 3). Persistent air leakage was found in 2 of 9 patients (22\%) with adhesions and 13 of $25(52 \%)$ without adhesions ( $p=0.37$, Fisher's exact test). Neither duration of preoperative drainage nor presence of persistent air leakage was associated with the incidence of adhesions.

The duration of operation ranged from 30 to 96 minutes (mean, 54 minutes) in patients with adhesion and from 31 to 139 minutes (mean, 62 minutes) in those without adhesions, also indicating no significant difference $(p=0.37$.

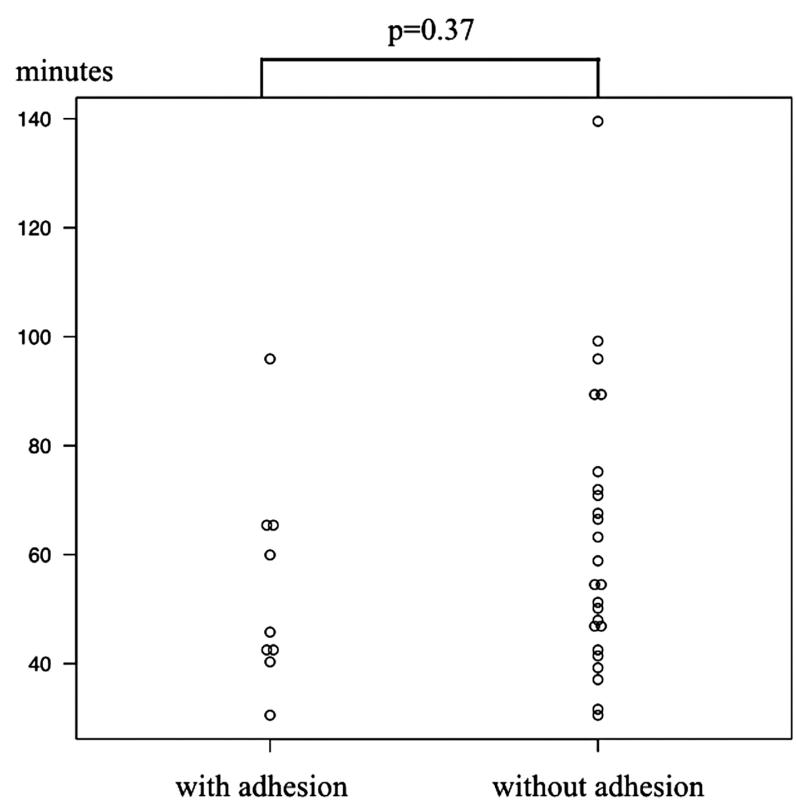

Fig. 4 Distribution of operation duration. There is no statistically significant difference between patients with and without adhesions.

Mann-Whitney U-test) (Fig. 4). The presence of adhesions due to preoperative drainage was not associated with the duration of operation.

\section{Discussion}

The use of the Thoracic Vent for outpatient drainage therapy in patients with spontaneous, traumatic or iatrogenic pneumothorax was first reported in 1991.5) The Thoracic Vent is currently used primarily in patients with spontaneous pneumothorax..$^{2)}$ Drainage therapy with the Thoracic Vent is beneficial for pneumothorax patients because it can be performed in an outpatient setting, making hospitalization unnecessary. Because primary spontaneous pneumothorax often occurs in students and young workers, it is important to avoid unscheduled admissions.

We inserted the Thoracic Vent under local anesthesia and did not usually change the drape or sterilize during drainage. With this management approach, we did not experience infections related to the Thoracic Vent although infection was previously reported. ${ }^{9)}$ We usually assessed lung status by X-ray twice or three times a week, and removed the Thoracic Vent when the lung fully expanded and air leakage stopped. Patients who were scheduled to undergo surgery were generally admitted 1 day preoperatively as scheduled. With this 
management approach, the total cost of drainage therapy was reduced, but the cost of a single day's hospitalization increased. ${ }^{2)}$ Thus, the Thoracic Vent is cost-effective for both patients and hospitals.

Re-expansion lung edema has been reported as a complication of outpatient drainage with the Thoracic Vent. ${ }^{10)}$ Therefore, we excluded patients with severe pneumothorax; for instance, those who require oxygen inhalation and those with tachycardia or low blood pressure. Although Karasaki reported that outpatient drainage therapy is indicated for patients aged 50 years or younger, we previously reported that it is also safe in patients over age 50 years. ${ }^{1)}$

Some patients were cured by outpatient drainage therapy alone, whereas others required surgical intervention for prolonged air leakage or underwent bullectomy with the hope of preventing pneumothorax recurrence. ${ }^{1)}$ This study showed that preoperative outpatient drainage with the Thoracic Vent was useful for pneumothorax patients who underwent surgery, and did not affect either intraoperative or postoperative outcomes.

Outpatient drainage therapy aims to avoid immediate hospital admission for chest drainage. In this study, with the exception of one individual who was admitted due to massive air leakage, all patients were admitted for surgery as scheduled and were able to avoid hospitalization for drainage. Thus, outpatient drainage therapy is useful in patients who plan to undergo surgery for pneumothorax.

In 9 of 34 cases $(26 \%$ ), adhesions were observed intraoperatively between the parietal and visceral pleura around the Thoracic Vent. In all cases, the adhesions were loose and could be dissected bluntly. As a consequence, there was no difference in operation duration between patients with and without adhesions. In Thoracic Vent drainage durations of less than 3 weeks, there were no adhesions that led to surgical complications. In this study, there were no differences between patients with and without adhesions in terms of the duration of drainage and the presence of persistent air leakage. We could not identify any patient characteristics that increased the likelihood of adhesion formation during outpatient drainage.

Previous papers have reported that outpatient drainage therapy is useful because many pneumothorax patients can be cured by outpatient drainage alone.,46) Our data show that outpatient drainage therapy is also useful as a bridge to surgery. Generally, the indication for surgery in pneumothorax patients is decided during drainage. ${ }^{7,10)}$
Using outpatient drainage therapy, this decision can be made on an outpatient basis.

\section{Conclusion}

Outpatient drainage therapy with the Thoracic Vent was useful in patients with spontaneous pneumothorax who subsequently underwent surgery, and when conducted for less than 3 weeks, this outpatient treatment did not affect intraoperative or postoperative outcomes.

\section{Disclosure Statement}

All authors have no conflict of interest.

\section{References}

1) Sano A, Yotsumoto T, Tsuchiya T. Outpatient drainage for patients with spontaneous pneumothorax over 50 years of age. Lung India 2017; 34: 232-5

2) Tsuchiya T, Sano A. Outpatient treatment of pneumothorax with a thoracic vent: economic benefit. Respiration 2015; 90: 33-9.

3) Sano A, Tsuchiya T, Nagano M. Outpatient drainage therapy with a thoracic vent for traumatic pneumothorax due to bull attack. Korean J Thorac Cardiovasc Surg 2014; 47: 563-5.

4) Karasaki T, Shintomi S, Nomura $Y$, et al. Outcomes of outpatient treatment for primary spontaneous pneumothorax using a small-bore portable thoracic drainage device. Thorac Cardiovasc Surg 2014; 62: 516-20.

5) Samelson SL, Goldberg EM, Ferguson MK. The thoracic vent. Clinical experience with a new device for treating simple pneumothorax. Chest 1991; 100: 880-2.

6) Martin T, Fontana G, Olak J, et al. Use of pleural catheter for the management of simple pneumothorax. Chest 1996; 110: 1169-72.

7) Yotsumoto T, Sano A, Sato Y. [Spontaneous pneumothorax during pregnancy successfully managed with a thoracic vent before surgical therapy; report of a case]. Kyobu Geka 2015; 68: 1031-3.

8) Kanda Y. Investigation of the freely available easyto-use software 'EZR' for medical statistics. Bone Marrow Transplant 2013; 48: 452-8.

9) Sakamoto K, Okita M, Tsuchida K. Outpatient management for spontaneous pneumothorax using truclose thoracic vent. Japan J Chest Surg 2001; 15: 18-22. (in Japanese)

10) Schoenenberger RA, Haefeli WE, Weiss P, et al. Timing of invasive procedures in therapy for primary and secondary spontaneous pneumothorax. Arch Surg 1991; 126: 764-6. 